\title{
Distribución de caudales de riego para las comunidades de la UCICMA - Imbabura
}

\section{Irrigation water distribution in the communities of the UCICMA - Imbabura}

\author{
Pablo Miguel Andrango Qimbiamba ${ }^{1}$, Randon Stalin Ortiz Calle ${ }^{1}$, Yessenia Gissella Cuaspa Ortega ${ }^{1}$ \\ $凶$ Autor para correspondencia: andrangomiguel@gmail.com \\ ${ }^{1}$ Universidad Central del Ecuador, Facultad de Ciencias Agricolas, Carrera Ingeniería Agronómica, Jerónimo Leiton y Av. La Gasca s/n, \\ Ciudadela Universitaria. Quito. 170521. Ecuador
}

\begin{abstract}
Resumen
La distribución del agua de riego en función del área cultivada y las necesidades hídricas de los cultivos es importante en los procesos de socialización y debate para generar la distribución equitativa del agua entre las comunidades indígenas del Ecuador. El presente estudio consistió en determinar el área máxima de riego considerando los requerimientos hídricos de los cultivos y el caudal concesionado, con el fin de proponer una alternativa de distribución del agua de riego entre las 12 comunidades que conforman la Unión de Comunidades de Indígenas y Campesinos de Mariano Acosta (UCICMA). Para el cálculo de la necesidad hídrica de los cultivos se utilizó la siguiente metodología: evapotranspiración potencial (Penman Monteith), precipitación efectiva (SCS), coeficiente de cultivo (FAO56), áreas de cultivo, fechas de siembra, ciclo vegetativo (mediante encuestas) e información de suelos (a partir de estudios realizados en la zona). Los resultados principales del estudio son: i) el caudal ficticio continuo ponderado es de $0,58 \mathrm{l} \mathrm{s}^{-1} \mathrm{ha}^{-1}$; ii) para irrigar la superficie total de las 12 comunidades se requiere un caudal de $950,551 \mathrm{~s}^{-1}$; iii) considerando el caudal concesionado de 3601 $\mathrm{s}^{-1}$, el área máxima técnicamente a irrigarse es 619,96 ha. La conclusión principal del estudio es que el caudal concesionado de $3601 \mathrm{~s}^{-1}$ permitirá cubrir el riego de 619,96 ha, correspondiente a $37 \%$ del área potencialmente regable de la UCICMA.
\end{abstract}

Palabras clave: Ecuador, balance hídrico, necesidades hídricas de los cultivos, área potencial de riego, caudal continuo.

\begin{abstract}
The distribution of irrigation water according to the cultivated area and the crop water requirements is important in the processes of equitable distribution of water among the indigenous communities of Ecuador among socialization and debate within the population. The present research consisted of determining the maximum area of irrigation according to the crop water requirements and the discharge delivered. With this data the research presents an alternative for water distribution for irrigation among the 12 communities of Unión de Comunidades Indígenas y Campesinos of Mariano Acosta (UCICMA). To calculate the crop water requirements, the following methodology was used: potential evapotranspiration (Penman Monteith), effective precipitation (SCS), crop coefficient (FAO 56), cultivation areas, planting dates and vegetative cycle (by surveys) and soil information (from studies conducted in the area). The main results of the study are: i) the continuous fictitious flow rate was about $0.581 \mathrm{~s}^{-1} \mathrm{ha}^{-1}$; ii) to irrigate the total area of the 12 communities a discharge of $950.551 \mathrm{~s}^{-1}$ is
\end{abstract}


required; iii) considering the discharge assigned of $3601 \mathrm{~s}^{-1}$, the maximum area that can be technically irrigated is about $619.96 \mathrm{ha}$. The main conclusion of the study is that the assigned discharge of $360 \mathrm{l} \mathrm{s}^{-1}$ will cover an irrigation area of 619.96 ha, corresponding to $37 \%$ of the potential irrigable area of the UCICMA.

Keywords: Ecuador, water balance, crop water requirements, potential irrigation area, continuous discharge.

\section{Introducción}

El riego es un factor muy importante en la producción de los cultivos, pues le permite al agricultor mejorar su calidad de vida. El desafío constante de los profesionales del agro es conocer la interacción entre el clima, el suelo, la genética vegetal y el manejo cultural, para mejorar la eficiencia del uso del agua (Micucci \& Álvarez, 2005).

La viabilidad de un proyecto de riego está íntimamente relacionada con factores productivos, de ingeniería, sociales, económicos y ambientales; por tanto, la cuantificación de cada uno de estos recursos es de vital importancia (García, 1996). Dentro de estos recursos, la cuantificación de los requerimientos hídricos de los cultivos constituye la columna vertebral de todo sistema de riego, siendo el parámetro básico y fundamental para la planificación de los sistemas de irrigación (Ortiz, 2011), puesto que permite diseñar reservorios, dimensionar redes de riego, sean estas a cielo abierto (canales) o presurizadas, estaciones de bombeo, programar el riego dentro de la parcela, planificar la distribución de los caudales en los ramales principales y secundarios, así como, definir los turnos de riego y la operación del sistema (Ortiz, 2011).

Los requerimientos de riego de los cultivos dependen de la altura sobre el nivel medio del mar, clima, suelos, tipo y ciclo vegetativo del cultivo, necesidades de lavado, eficiencia del sistema de riego, de las prácticas culturales y agronómicas empleadas durante el ciclo de producción de los cultivos (Ortiz, 2011).

El directorio de la Unión de Comunidades de Indígenas y Campesinos de Mariano Acosta (UCICMA) busca la generación de oportunidades de desarrollo a través del riego a fin de evitar la migración de la población joven hacia las ciudades, para conservar y valorizar el territorio (IEDECA, 2015). La organización cuenta con una superficie cultivable de 1.666,63 hectáreas y con un caudal concesionado de $3601 \mathrm{~s}^{-1}$. Los estudios socio económicos previos, junto al presente, permitirán elaborar propuestas de distribución del agua equitativas entre las 12 comunidades que la conforman (IEDECA, 2016).

Con estos antecedentes, la pregunta que se planteó para el desarrollo de la presente investigación fue: ¿el caudal concesionado es suficiente para irrigar el área total cultivable? El objetivo del estudio fue determinar el área de riego máxima a partir del caudal concesionado para proponer una alternativa de distribución del agua con base en las necesidades hídricas de los cultivos y el área de riego.

\section{Materiales y métodos}

\section{1. Área de estudio}

La UCICMA está ubicada en la provincia de Imbabura, República del Ecuador; fue creada en 1989 y legalizada en 2005. Se encuentra conformada por 14 comunidades filiales que pertenecen a las parroquias de Pimampiro (8 comunidades), Mariano Acosta (4 comunidades) y Ambuquí (2 comunidades) (CIPRADEC, 2014). Las 14 comunidades están conformadas por 552 familias. En la investigación se trabajó con 12 comunidades dado que las dos comunidades restantes decidieron no participar en el proceso.

La comunidad se encuentra ubicada a una altitud comprendida entre 1.920 y 3.520 m.s.n.m.; a una latitud de $00^{\circ} 03^{\prime} 23^{\prime \prime} \mathrm{S}$ y una longitud de $-77^{\circ}$ 98'33' O (Figura 1). El clima de la zona se caracteriza por tener dos épocas bien diferenciadas hidrológicamente: una época lluviosa o invierno (de octubre a abril) y una época seca o de verano (de mayo a septiembre). La precipitación anual promedio del proyecto es de $813,26 \mathrm{~mm}$ y la evapotranspiración de referencia anual promedio de $1.040,70 \mathrm{~mm}$. 


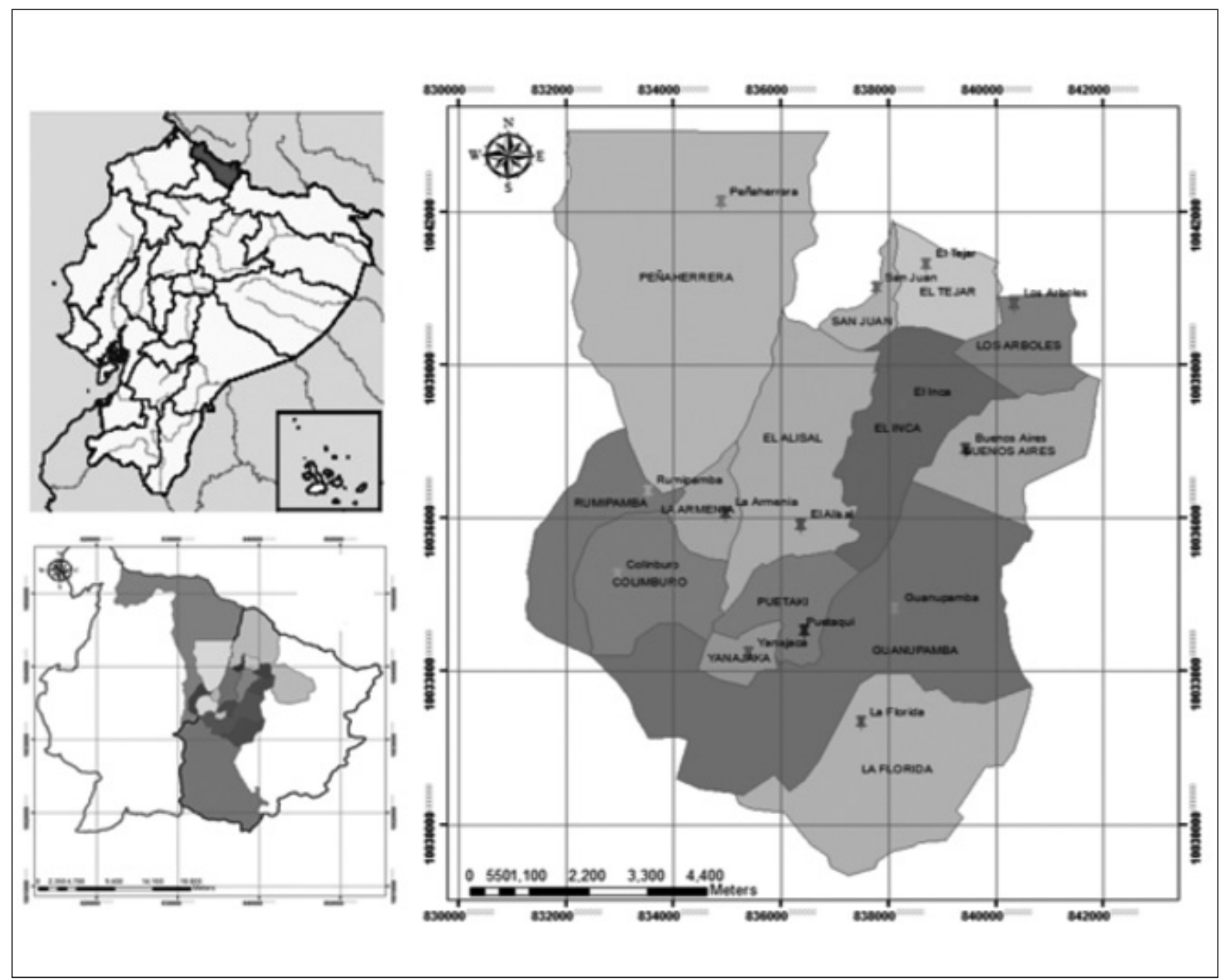

Figura 1. Ubicación de la UCICMA.

\subsection{Materiales}

Para la elaboración del proyecto se utilizó la siguiente información: cartografía de la zona del proyecto; información climatológica de las estaciones: Atuntaqui, Olmedo, San Gabriel, Mira, San Vicente de Pusir, Ambuquí, Monte Olivo, Pimampiro, Ibarra, Mariano Acosta, Zuleta y San Francisco de Sigsipamba (INAMHI, 2013); coeficientes de cultivo determinados con base en el Manual No. 56 de la FAO; datos de los suelos a partir de estudios e información de los cultivos; áreas, fechas de siembra a partir de encuestas.

\subsection{Metodología}

- Evapotranspiración de referencia. La evapotranspiración de referencia se determinó por el método Penman Monteith FAO56 (FAO 2006a, 2006b), para el cultivo de referencia con una altura de $12 \mathrm{~cm}$, bien regado y con una cobertura total de la superficie del suelo, la resistencia superficial estimada es de $70 \mathrm{~s} \mathrm{~m}^{-1} \mathrm{y}$ un albedo de 0,23 (Allen et al., 2006).

$$
E T_{o}=\frac{0,408 \times \Delta \times(R n-G)+\gamma \times \frac{900}{(T+273)} \times U_{2} \times(e s-e a)}{\Delta+\gamma \times\left(1+0,34 \times U_{2}\right)}
$$

Donde: $E T_{\mathrm{o}}$ es la evapotranspiración referencia $\left(\mathrm{mm} \mathrm{d}^{-1}\right) ; R n$, radiación neta en la superficie del cultivo ( $\left.\mathrm{MJ} \mathrm{m}^{-2} \mathrm{~d}^{-1}\right)$; $G$, flujo del calor de suelo $\left(\mathrm{MJ} \mathrm{m}^{-2} \mathrm{~d}^{-1}\right) ; U_{2}$, velocidad del viento medido a 2 metros de altura $\left(\mathrm{m} \mathrm{s}^{-1}\right)$; ea, presión real de vapor $(\mathrm{kPa})$; es, presión de vapor de saturación; 
$\Delta$, pendiente de la curva de variación de la presión $\left(\mathrm{kPa}{ }^{\circ} \mathrm{C}^{-1}\right) ; T$, temperatura promedio $\left({ }^{\circ} \mathrm{C}\right)$; $\gamma$, constante psicométrica $\left(\mathrm{kPa}^{\circ} \mathrm{C}^{-1}\right) ; Z$, es la elevación sobre el nivel medio del mar; $\lambda$, calor latente de vaporización $\left(\mathrm{MJ} \mathrm{kg}^{-1}\right)$. Para obtener la $\mathrm{ET}_{\mathrm{o}}$ a nivel mensual se debe multiplicar el valor de $\mathrm{ET}_{\mathrm{o}}$ por el número de días del mes correspondiente.

- Evapotranspiración real. La evapotranspiración real del cultivo se determinó por el método de la FAO (Allen et al., 2006).

$$
E T r=K c \times E T o
$$

Donde: ETr, es la evapotranspiración real del cultivo mensual $\left(\mathrm{mm} \mathrm{mes}^{-1}\right) ; \mathrm{Kc}$, es el coeficiente del cultivo.

- Precipitación efectiva. La precipitación efectiva se determinó por el método del Servicio de Conservación de Suelos de los Estados Unidos a partir de la precipitación con una probabilidad de ocurrencia del 75\% (Ortiz, 2011).

$$
P e=f \times 10^{0,00095 \times E T o}\left(1,2525 \times P^{0,8242}-2,9352\right)
$$

$$
f=0,5317+0,0116 \times L A A-0,000089 \times L A A^{2}+0,00000023 \times L A A^{3}
$$

Donde: $P e$, es la precipitación efectiva $(\mathrm{mm}$ $\left.\mathrm{mes}^{-1}\right) ; P$, es la precipitación con una probabilidad de ocurrencia del $75 \%$; $L A A$, es la lámina de agua aprovechable $\left(\mathrm{mm} \mathrm{mes}^{-1}\right) ; f$, es el factor de ajuste de la precipitación efectiva en función del agua disponible en el suelo.

- Láminas de agua aprovechable y neta. A partir de las constantes hidrofísicas de los suelos se determinaron las láminas de agua aprovechable y neta (Ortiz, 2011).

$$
\begin{aligned}
& L A A=\frac{C C-M P}{100} \times \operatorname{Pr} \\
& L n=p \times L A A
\end{aligned}
$$

Dónde: $C C$, es el contenido de humedad en términos de volumen de capacidad de campo (\%);
$M P$, es el contenido de humedad en términos de volumen de marchitez permanente (\%); $P r$, es la profundidad radicular efectiva $(\mathrm{mm}) ; \mathrm{Ln}$, es la lámina neta o el agotamiento máximo permitido del agua en el suelo (mm); $p$, es el porcentaje de agotamiento, típico para cada cultivo (decimal).

- Isolíneas de ET y Pe. Con la ayuda de sistemas de información geográfica, para cada mes se trazaron las isolíneas de $\mathrm{ET}_{0}$ y Pe, las cuales permitieron determinar $\mathrm{ET}_{\mathrm{o}} \mathrm{y}$ Pe para cada comuna.

- Patrón de cultivos. Una vez procesada la información de las encuestas se procedió a elaborar el patrón de cultivos (de ciclo corto, anual y perenne) para cada comunidad.

- Coeficiente de cultivo. Los coeficientes de cultivo $(\mathrm{Kc})$, se determinaron a partir de los coeficientes de las fases propuestas por la FAO en el Manual Técnico No. 56 (Allen et al., 2006).

- Balance hídrico. A nivel mensual, para cada comuna y para cada cultivo, se determinó el balance hídrico por el método del balance de masas, en donde un valor positivo refleja un exceso y un valor negativo un déficit hídrico, identificándose este valor como NHM (Ortiz, 2011).

$$
N H M=P e+D H-E T r
$$

Donde: NHM, necesidad hídrica mensual (mm); DH, es el almacenamiento de la humedad en el suelo (mm).

- $\quad$ Necesidad hídrica diaria. A partir de la necesidad hídrica mensual, se determinó la necesidad hídrica diaria por el método desarrollado por el servicio de conservación de suelos de los Estados Unidos (Ortiz, 2011).

$$
N H D=0,034 \times \frac{N H M^{1,09}}{L n^{0,09}}
$$

Donde: $N H D$, es la necesidad hídrica diaria $\left(\mathrm{mm} \mathrm{d}^{-1}\right)$. 
- Caudal ficticio continuo. El caudal ficticio continuo se determinó a partir de la necesidad hídrica diaria y es el caudal que se requiere suministrar a una superficie de una hectárea en forma continua durante las 24 horas del día para cubrir la demanda evaporativa de la atmósfera (Ortiz, 2011).

$$
q f c=\frac{N H D}{8,64}
$$

Donde: $q f$, es el caudal ficticio continuo $\left(1 \mathrm{~s}^{-1} \mathrm{ha}^{-1}\right)$.

- Caudal de riego en parcela. Es el caudal que se requiere en cabecera de parcela, en función del caudal ficticio continuo, la eficiencia del método de aplicación del agua, tiempo de riego diario y el período de riego dentro de la frecuencia de riego (Ortiz, 2011).

$$
Q r p=\frac{q f c}{E f a} \times \frac{24}{T r d} \times \frac{F R}{P R}
$$

Donde: Qrp, es el caudal de riego en parcela (1 $\left.\mathrm{s}^{-1} \mathrm{ha}^{-1}\right) ; E f a$, es la eficiencia de aplicación del método de riego (decimal); $T r d$, es el tiempo de riego diario (horas); $F R$, es la frecuencia de riego (días); $P R$, es el período de riego (días). Para este proyecto, Trd fue de 24 horas, FR de 7 días, PR de 7 días y Efa de 0,7 (riego por aspersión).

- Área a irrigarse. El área máxima de riego se determinó al dividir el caudal concesionado para el caudal de riego en parcela.
- Factor de ajuste para la distribución del agua. El factor de ajuste global para distribuir el agua se obtuvo a partir de dividir el área máxima de riego para la superficie potencial de riego de la UCICMA. Este factor de ajuste no considera aspectos sociales, distribución de la tierra, agricultores dedicados a la agricultura, índice de pobreza, entre otros.

- Caudal de entrega para cada comunidad. Se determinó al multiplicar el factor de ajuste por la superficie de cada comuna y por el caudal de riego en parcela.

- Encuesta. No existe norma estadística que se pueda aplicar a este tema, debido a que se debe levantar información de campo (sistemas de producción). Por tal motivo, la encuesta se realizó a más del 50\% de los agricultores (Ortiz, 2011).

\section{Resultados y discusión}

\subsection{Datos generales de superficie}

La superficie total potencialmente irrigable en la UCICMA fue de 1.666,36 hectáreas y se requiere un caudal de 950,55 $1 \mathrm{~s}^{-1}$ para irrigarla. La superficie que se puede irrigar técnicamente para cubrir las necesidades hídricas de los cultivos a partir del caudal concesionado es de $3601 \mathrm{~s}^{-1}$ fue de 619,96 hectáreas (Figura 2).

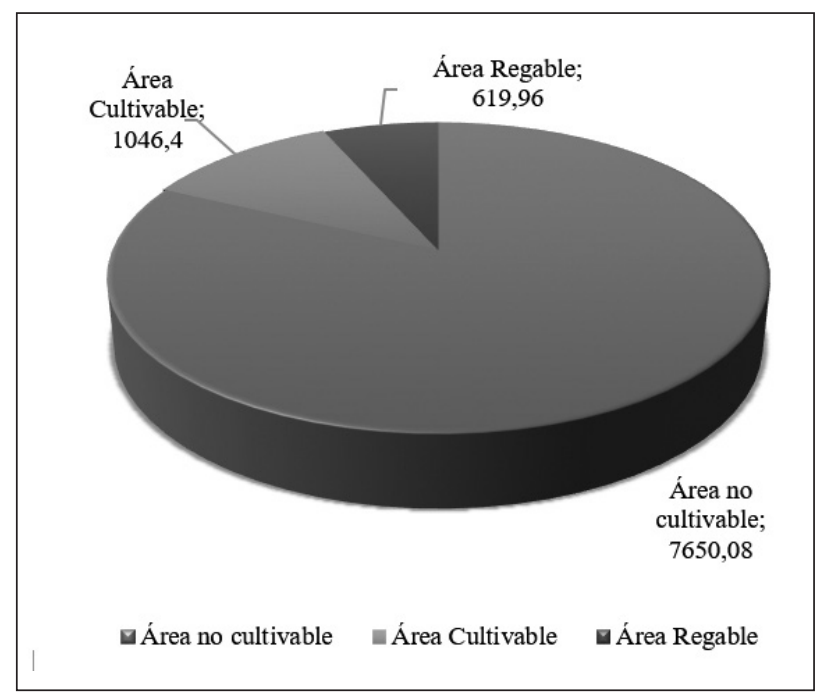

Figura 2. Superficie total de la UCICMA. 


\subsection{Patrón de cultivos}

Se determinó una superficie cultivada de 810,09 hectáreas, distribuidas conforme la Tabla 1.

Tabla 1. Superficie cultivada

\begin{tabular}{|c|c|c|c|}
\hline Cultivos & $\begin{array}{c}\text { Área } \\
\text { ha }\end{array}$ & $\begin{array}{c}\text { NHD } \\
\text { mm d }^{-1}\end{array}$ & $\begin{array}{c}\text { qfe } \\
\mathrm{ls}^{-1} \mathrm{ha}^{-1}\end{array}$ \\
\hline Aguacate & 36,78 & 1,81 & 0,21 \\
\hline Ají & 1,00 & 2,67 & 0,31 \\
\hline Alfalfa & 0,25 & 2,43 & 0,28 \\
\hline Arveja & 120,00 & 2,33 & 0,27 \\
\hline Babaco & 1,30 & 2,26 & 0,26 \\
\hline Cebada & 19,69 & 2,04 & 0,24 \\
\hline Cebolla larga & 0,55 & 2,77 & 0,32 \\
\hline Cebolla paiteña & 2,25 & 2,26 & 0,26 \\
\hline Chocho & 2,75 & 3,28 & 0,38 \\
\hline Reina claudia & 1,24 & 2,41 & 0,28 \\
\hline Durazno & 8,77 & 2,05 & 0,24 \\
\hline Fréjol & 41,95 & 3,54 & 0,41 \\
\hline Granadilla & 2,20 & 2,81 & 0,33 \\
\hline Haba & 17,07 & 2,11 & 0,24 \\
\hline Hortalizas & 1,10 & 2,47 & 0,29 \\
\hline Limón & 6,55 & 1,40 & 0,16 \\
\hline Maíz & 135,30 & 2,43 & 0,28 \\
\hline Mandarina & 6,02 & 1,40 & 0,16 \\
\hline Manzana & 1,89 & 1,95 & 0,23 \\
\hline Melloco & 0,35 & 2,59 & 0,30 \\
\hline Mora de castilla & 4,45 & 2,19 & 0,25 \\
\hline Naranja & 3,45 & 2,14 & 0,25 \\
\hline Naranjilla & 0,50 & 3,38 & 0,39 \\
\hline Ocas & 0,75 & 2,10 & 0,24 \\
\hline Papa & 41,63 & 2,57 & 0,30 \\
\hline Pasto mixto & 273,40 & 2,46 & 0,29 \\
\hline Pera & 0,33 & 2,67 & 0,31 \\
\hline Pimiento & 0,50 & 2,67 & 0,31 \\
\hline Quinua & 7,00 & 3,13 & 0,36 \\
\hline Taxo & 2,81 & 3,28 & 0,38 \\
\hline Tomate de árbol & 7,79 & 1,54 & 0,18 \\
\hline $\begin{array}{l}\text { Tomate riñón } \\
\text { (invernadero) }\end{array}$ & 20,67 & 2,89 & 0,33 \\
\hline $\begin{array}{l}\text { Tomate riñón } \\
\text { (campo abierto) }\end{array}$ & 7,28 & 2,65 & 0,31 \\
\hline Trigo & 26,65 & 2,77 & 0,32 \\
\hline Uvilla & 4,50 & 2,58 & 0,30 \\
\hline Zanahoria & 1,25 & 1,68 & 0,19 \\
\hline
\end{tabular}


El cultivo que cubre una mayor superficie es el pasto (mixto), con 273,48 hectáreas (33,76\%), el cultivo perenne de mayor superficie fue el aguacate con 36,78 hectáreas $(4,54 \%)$ y el cultivo de ciclo corto con mayor superficie fue el maíz con 135,33 ha (16,71\%), alrededor del 9\% del área cultivada son frutales (durazno, reina claudia, manzanas, etc.) y el $2 \%$ corresponde a cítricos (mandarina, limón naranja, etc.).

\subsection{Requerimientos hídricos de los cultivos}

\subsubsection{Demanda hídrica de los cultivos}

La demanda hídrica máxima se registró para el cultivo de fréjol con $3,54 \mathrm{~mm} \mathrm{~d}^{-1}$, equivalente a un caudal ficticio continuo de $0,411^{-1} \mathrm{~s}^{-1} \mathrm{y}$ el valor menor para el cultivo de limón, con $1,40 \mathrm{~mm} \mathrm{~d}^{-1}$, equivalente a un caudal ficticio continuo de 0,16 1 $\mathrm{s}^{-1} \mathrm{ha}^{-1}$. El caudal ficticio continuo ponderado fue de $0,581 \mathrm{~s}^{-1} \mathrm{ha}^{-1}$.
El caudal de riego en parcela (Qrp), considerando la operación del sistema durante las 24 horas del día y un periodo de riego igual a la frecuencia de riego de siete días, para el cultivo de naranjilla fue de $0,581 \mathrm{~s}^{-1} \mathrm{ha}^{-1} \mathrm{y}$ para el cultivo de limón de $0,231 \mathrm{~s} \mathrm{~s}^{-1} \mathrm{ha}^{-1}$.

\subsubsection{Demanda estacional}

La demanda estacional es la cantidad de agua que se requiere para cubrir la demanda hídrica de los cultivos durante los 12 meses del año. El área máxima de riego considerando el caudal concesionado de $3601 \mathrm{~s}^{-1}$ fue de 619,96 hectáreas, siendo el factor de ajuste de 0,37. La máxima demanda de agua se registró en los meses de enero y febrero, y la menor demanda en los meses de abril y noviembre (Figura 3). El caudal concesionado es constante a lo largo del año.

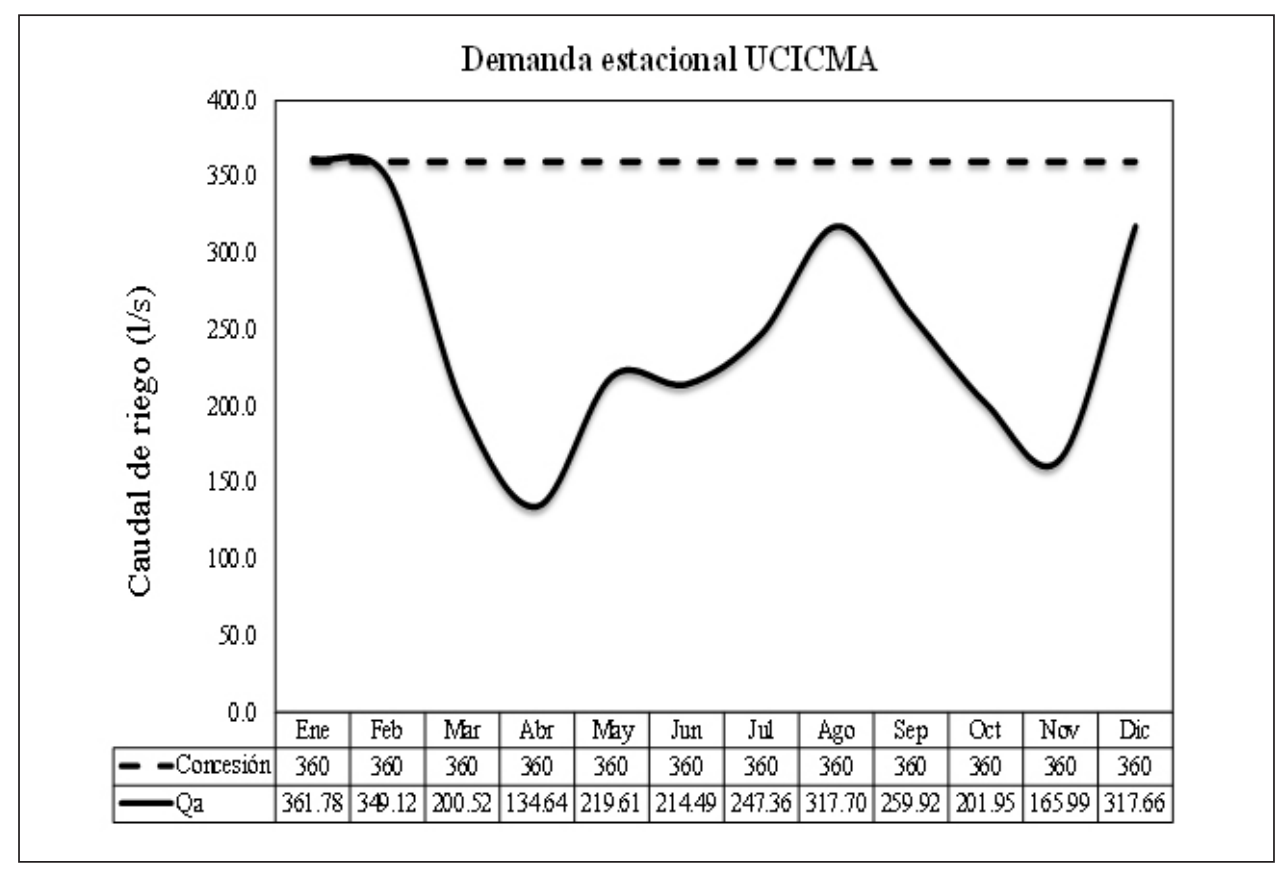

Figura 3. Demanda estacional.

\subsection{Propuesta de distribución del caudal}

La comunidad de Guanupamba demanda la mayor cantidad de agua $\left(87,041 \mathrm{~s}^{-1}\right)$ y Colimburo es la comunidad que demanda la menor cantidad de agua $\left(6,481 \mathrm{~s}^{-1}\right)$. La distribución planteada (Tabla 2), se realizó con base en la superficie que puede irrigarse técnicamente para cubrir las necesidades hídricas de los cultivos, considerando un ajuste equitativo a partir del caudal concesionado; este ajuste no toma en cuenta aspectos sociales como: índice de pobreza, minifundios, comunidades dedicadas a la agricultura, organización social, entre otros factores.

La limitante encontrada en el presente estudio es la concesión otorgada por la Secretaría Nacional del Agua, la cual no permitirá cubrir la demanda hídrica de las tierras potencialmente agrícolas o cultivables 
de la UCICMA. La curva de demanda estacional (Figura 3) muestra que, desde marzo hasta noviembre, el caudal concesionado puede almacenarse en reservorios para incrementar la superficie de riego.

Tabla 2. Caudales para cada comunidad

\begin{tabular}{|c|c|c|c|}
\hline Comunidad & $\begin{array}{c}\text { Área } \\
\text { cultivable } \\
\text { ha }\end{array}$ & $\begin{array}{c}\text { Área a irrigarse } \\
\text { ha }\end{array}$ & $\begin{array}{c}\text { Caudal } \\
1 \mathrm{~s}^{-1}\end{array}$ \\
\hline Los Árboles & 195,00 & 72,55 & 42,13 \\
\hline El Inca & 95,00 & 35,34 & 20,52 \\
\hline San Juan & 68,00 & 25,30 & 14,69 \\
\hline El Tejar & 37,33 & 13,89 & 8,06 \\
\hline Rumipamba & 216,95 & 80,71 & 46,87 \\
\hline Peñaherrera & 229,82 & 85,50 & 49,65 \\
\hline Guanupamba & 402,89 & 149,89 & 87,04 \\
\hline Puetaqui & 137,15 & 51,03 & 29,63 \\
\hline Yanajaca & 30,12 & 11,21 & 6,51 \\
\hline El Alisal & 55,00 & 20,46 & 11,88 \\
\hline Colimburo & 30,00 & 11,16 & 6,48 \\
\hline La Florida & 169,10 & 62,91 & 36,53 \\
\hline Total & 1666,36 & 619,96 & 360,00 \\
\hline
\end{tabular}

En el debate social para alcanzar los acuerdos sobre la distribución del agua, se recomienda considerar los siguientes aspectos en orden de importancia: i) agricultores dedicados a la agricultura; ii) minifundios; iii) índice de pobreza, y iv) organización social.

\section{Conclusiones}

La presente investigación permitió obtener las siguientes conclusiones:

- El caudal de riego máximo se registró en los cultivos de fréjol con $0,581 \mathrm{~s}^{-1} \mathrm{ha}^{-1}$, seguido del cultivo de tomate riñón bajo invernadero con $0,53 \mathrm{l} \mathrm{s}^{-1} \mathrm{ha}^{-1}$; los cultivos con la menor demanda fueron: habas con $0,161 \mathrm{~s}^{-1} \mathrm{ha}^{-1} \mathrm{y}$ el cultivo de tomate de árbol con $0,261 \mathrm{~s}^{-1} \mathrm{ha}^{-1}$.
- La superficie potencialmente irrigable fue de 1.666,36 hectáreas y se requiere un caudal de 950,55 $1 \mathrm{~s}^{-1}$ para cubrir la demanda de agua de los cultivos; la superficie máxima posible de irrigarse técnicamente fue de 619,36 hectáreas considerando el caudal concesionado de $3601 \mathrm{~s}^{-1}$.

- La distribución del caudal concesionado para las comunidades fue la siguiente: Los Árboles con 42,13 $1 \mathrm{~s}^{-1}$, El Inca con 20,52 $1 \mathrm{~s}^{-1}$, San Juan con $14,691 \mathrm{~s}^{-1}$, El Tejar con 8,06 $1 \mathrm{~s}^{-1}$, Rumipamba con 46,87 1 s ${ }^{-1}$, Peñaherrera con 49,65 1 $\mathrm{s}^{-1}$, Guanupamba con 87,04 1 s ${ }^{-1}$, Puetaqui con $29,631_{1 ~ s^{-1}}$, Yanajaca con $6,511 \mathrm{~s}^{-1}$, El Alisal con $11,881 \mathrm{~s}^{-1}$, Colimburo con $6,481 \mathrm{~s}^{-1}$ y La Florida con $36,531 \mathrm{~s}^{-1}$. 


\section{Referencias}

Allen, R., Pereira, L., Raes, D., \& Smith, M. (2006). Evapotranspiración del cultivo. Manual Técnico No. 56. Roma: FAO.

Corporación para el Desarrollo Social Económico y Productivo Cía. Ltda., CIPRADEC. (2014). Plan de Desarrollo y Ordenamiento Territorial del Cantón San Pedro de Pimampiro. Pimampiro, Ecuador: GAD. San Pedro de Pimampiro.

FAO (2006a). Evapotranspiración del cultivo. Obtenido de: www.fao.org/home/es/

FAO (2006b). Evapotranspiración del cultivo. Guías para la determinación de los requerimientos de agua de los cultivos. Obtenido de: http://www. fao.org/3/a-x0490s.pdf

García, J. (1996). Redes colectivas, determinación de caudales. In Sistema de riego por aspersión (p. 45). Madrid: Centro de Estudios y Experimentación de Obras Públicas (CEDEX).
Instituto de Ecología y Desarrollo de las Comunidades Andinas, IEDECA. (2015). Diagnóstico de sistemas de producción de las comunidades de Mariano Acosta. Ibarra-Ecuador: IEDECA.

Instituto de Ecología y Desarrollo de las Comunidades Andinas, IEDECA. (2016). Estudio de dinámicas productivas de las comunidades de la UCICMA. Ibarra-Ecuador: IEDECA.

Instituto Nacional de Meteorología e Hidrología , INAMHI. (2013). Estaciones meteorológicas. Obtenido de: http://www.serviciometeorologico.gob.ec/

Micucci, F., \& Álvarez, C. (2005). El agua en la produccion de cultivos extensivos. III. Impacto de las prácticas de manejo sobre la eficiencia de uso del agua. INPOFOS Archivo Agronómico, 8, 1-4.

Ortiz, R. (2011). Sistema de riego Podocarpus Yangana: requerimientos de riego y planificación del sistema. Loja, Ecuador. 